\title{
Effects of social restrictions on people with dementia and carers during the pre-vaccine phase of the COVID-19 pandemic: experiences of IDEAL cohort participants
}

Claire Pentecost ( $\square$ c.pentecost@exeter.ac.uk)

University of Exeter https://orcid.org/0000-0003-2048-5538

Rachel Collins

University of Exeter https://orcid.org/0000-0002-3405-7932

\section{Sally Stapley}

University of Exeter https://orcid.org/0000-0001-5538-8717

\section{Christina Victor}

Brunel University London https://orcid.org/0000-0002-4213-3974

\section{Catherine Quinn}

University of Bradford https://orcid.org/0000-0001-9553-853X

\section{Alexandra Hillman}

Swansea University https://orcid.org/0000-0003-1859-1075

Rachael Litherland

Innovations in Dementia CIC

\section{Louise Allan}

University of Exeter https://orcid.org/0000-0002-8912-4901

\section{Linda Clare}

University of Exeter https://orcid.org/0000-0003-3989-5318

\section{Research Article}

Keywords: Alzheimer's disease, carers, pandemic, vaccine, qualitative analysis

Posted Date: February 28th, 2022

DOI: https://doi.org/10.21203/rs.3.rs-1403858/v1

License: (c) (1) This work is licensed under a Creative Commons Attribution 4.0 International License. Read Full License 


\section{Abstract}

This qualitative study was designed to understand the impact of social distancing measures on people with dementia and carers living in the community during a period of ongoing restrictions before the COVID-19 vaccination roll-out in England and Wales. We conducted semi-structured interviews with 11 people with dementia and 10 carers (including 3 dyads) living in the community in England and Wales. Participants were recruited during November and December 2020. We used framework analysis to identify issues and elicit suggestions for potential solutions.

We identified three interrelated themes. People with dementia experienced a fear of decline in capabilities or mood and attempted to mitigate this. Carers were aware of changes in the person with dementia and an increase in caring responsibilities and for some, there was a change in the relationship. Subsequently, reduced confidence in capabilities to navigate a new and hostile environment created a cyclical dilemma of returning to 'normal' where not returning to usual activities made things worse. People with dementia and carers had feelings of neglect and being alone in their struggle, alongside feeling socially excluded during the pandemic when comparing themselves to others in society, and there was little optimism associated with the upcoming vaccine programme.

People found their own solutions to reduce the effects of isolation by keeping busy and socially active and practising skills deemed to help reduce the progression of dementia. This and some limited local public initiatives for the general public facilitated feelings of social inclusion. This study adds understanding to existing evidence about the longer-term experience of social isolation several months into the pandemic highlighting the importance of health and community groups and how services can find ways to support, include and interact with people with dementia and carers during and after social restrictions.

\section{What Is Known About This Topic}

- Social restrictions to manage COVID-19 infections had negative impacts on cognitive functioning and mood for people with dementia early in the pandemic.

- Carers struggled with the temporary closure of dementia services and associated respite.

\section{What This Paper Adds}

- People with dementia and carers did not feel they could return to previous activities when things reopened, and this did not change when the vaccination programme was announced.

- A further decrease in confidence and perceived decline in people with dementia was a barrier to returning to usual activities.

- People with dementia attempted to minimise decline and to stay socially engaged, but they, and carers felt they did this alone and felt socially excluded during the pandemic. 


\section{Introduction}

The enforced social distancing measures to minimise the transmission of COVID-19 resulted in restrictions in social contact, and for some, isolation for long periods. This has had psychological, social, and physical health consequences (Pongan et al., 2021). Social inclusion is important for the well-being of people with dementia (Pinkert et al. 2021) and their carers (Greenwood et al., 2018), and as a section of our society already recognised as marginalised (Wright and O'Connor (2018), they could suffer disproportionately.

International evidence has highlighted the complex difficulties experienced by people with dementia and carers living in the community related to social restrictions during the pandemic (Clemente-Suarez et al., 2021). Early surveys suggest that protracted isolation has triggered an increase in neuropsychiatric symptoms and functional decline for people with dementia (Borelli et al., 2021, Cohen et al., 2020, Ismail et al., 2021 Simonetti et al., 2020).

For carers, mental and physical health has deteriorated (Alzheimer's Society 2020, Masterson et al., 2021), influenced by the increased complexity and burden of care with little or no access to support or respite (Cagnin et al.,2020, Canevelli et al., 2020, Carpinelli et al., 2020, Giebel et al., 2021b, O'Rourke et al., 2021). The closure of services early in the pandemic caused concern about whether the person with dementia would be unable to return, due to progression of symptoms (Giebel et al., 2021a). Feelings of isolation have been greater for those unable to access the internet (O'Rourke et al., 2021a, Siefert et al., 2021).

Social isolation is a particular concern for people with dementia (Gardiner et al. 2020) due to the loss of social networks and support (Kane and Cook 2013). Social exclusion is a wider issue associated with discrimination and linked to deprivation and inequality and has implications for well-being in people with dementia (Wu et al. 2018). Social exclusion can change over time and exclusionary factors are multilevel, indicating individual circumstances and skills, and forces such as policy and social norms (Khan et al. 2015).

Many people with dementia are already vulnerable to the wider effects of social exclusion due to a complex range of factors including societal attitudes and health status (Pinkert et al., 2021), but during the pandemic, the risk of social exclusion could have increased. In their review of old-age social exclusion, Walsh et al. (2017) identified six interconnected common dimensions of social exclusion experienced by older people: civic participation; services, amenities and mobility; materials and financial resources; social relations; socio-cultural aspects; neighbourhood and community. Cultural/social and emotional dimensions including maintaining relationships have been particularly important for people with dementia (Pinkert et al., 2021, Quinn et al. 2021a, Quinn et al. 2021b). Reduced face-to-face interaction during the pandemic has made it difficult to conduct relationships (O'Rourke et al., 2020). It is not surprising therefore that social distancing measures and the closure of support services have led to increased feelings of social exclusion and abandonment amongst people with dementia and caregivers 
(Bascu 2021 et al., Clare et al., 2021, O’Rourke et al., 2021a, Giebel et al., 2021b, Rochford-Brennan et al., 2020).

This study was conducted when social restrictions were in force, but during a time later in the pandemic when restrictions were expected to ease due to the upcoming introduction of the vaccination programme in the UK. We collected interviews with people with dementia and carers living in the community during a period of lockdown eight months after the start of the pandemic (November 2020) and just before the roll-out of the vaccine programme in England and Wales in December 2020. We aimed to understand the longer-term and ongoing impact of social restrictions (lockdown) and to identify preferred strategies and supportive responses that could address impacts of social exclusion and assist recovery from being isolated.

\section{Methods}

\section{Design}

This study is a qualitative interview study using framework analysis to identify themes in participants' accounts (Gale et al., 2013).

\section{Recruitment and data collection}

Participants in the INCLUDE (Identifying and mitigating the individual and dyadic impact of COVID-19 and life under physical distancing on people with dementia and carers) study (Clare et al., 2021) were invited to take part. INCLUDE is embedded within the longitudinal IDEAL cohort study (Clare et al., 2014; Silarova et al., 2018). All cohort participants with dementia were community-dwelling and had mild to moderate dementia at the time of recruitment, and in each case, an informal (family) carer was invited to participate. IDEAL was approved by Wales Research Ethics Committee 5 (reference 13/WA/0405) and IDEAL-2 by Wales Research Ethics Committee 5 (reference 18/WS/0111) and Scotland A Research Ethics Committee (reference 18/SS/0037). INCLUDE was approved as an amendment to IDEAL-2 for England and Wales (18/WS/0111 AM12).

People with dementia and carers, either as individuals or dyads, who were participating in INCLUDE, and who had consent to be contacted for a follow-up interview ( $n=35$ individuals at the time of recruiting), were eligible. We telephoned 27 people before the vaccine roll-out was announced. We could not contact six. Only those living at home and able to provide informed consent for audio-recording over the telephone or via Zoom were included. We attempted to recruit similar numbers of males and females and carers and people with dementia and stopped recruiting when the vaccine roll-out was announced.

Interviewers aimed to elicit the experiences of participants from their perspective as someone with dementia, or a carer of someone with dementia. Interviews took place between 4th November 2020 and 1 st December 2020 when the numbers of COVID-19 cases were rising and England and Wales moved into the second strict 'lockdown' with restrictions on movement and activity to reduce the spread of the 
disease. Two researchers (CP, RC) conducted semi-structured interviews with no theoretical focus using a topic guide (Table 1) designed with input from the IDEAL Patient and Public Involvement (ALWAYS) group (Litherland et al. 2018).

Table 1

Topic Guide showing topics raised for each of the stages experienced during the pandemic

\section{Timeline}

Topic

Difficulties or changes to daily routines

Own coping strategies and or support found to be helpful.

Additional or missing support or information that might have been helpful.

Any unexpected benefits or outcomes; and how they might be maintained.

Training or information health or social care professionals or volunteers need to help people with memory difficulties in the COVID-19 situation

\section{Analysis}

All interviews were digitally audio-recorded and transcribed verbatim, anonymised, and uploaded to Nvivo12.

Two researchers (CP and $\mathrm{RC}$ ) familiarised themselves with all recordings and transcripts. An initial framework was developed by independently open-coding two carer transcripts and two transcripts from people with dementia, and comparing and revising until agreement was reached. The remaining transcripts were then coded the using the agreed framework and then organised into categories and themes. CP explored patterns and relationships between themes using charts, and by comparing themes and testing possible interpretations against the original data. CP discussed the emerging findings with RC and the research team to explore alternative interpretations.

\section{Findings}

\section{Participants}

Two researchers conducted 18 interviews representing 21 people: 11 people with dementia and 10 carers. Three of the interviews were conducted with dyads interviewed together and the members of one dyad (PD4 and C4) were interviewed separately. The other 13 interviews were with individuals not connected. Participant characteristics can be found in Table $2 a$ and Table $2 b$. 
Table 2

a Characteristics of participants with dementia

\begin{tabular}{|lllll|}
\hline ID & Age in years & Gender & Dementia Type & Living arrangements \\
\hline PD1 & 67 & M & AD & living with partner \\
\hline PD2 & 84 & F & AD & living alone \\
\hline PD3 J & 88 & F & AD & living with spouse \\
\hline PD4 & 64 & M & VaD & living with partner \\
\hline PD5 & 77 & M & AD & living alone \\
\hline PD6 & 67 & M & FTD & living with partner \\
\hline PD7 & 50 & F & AD & living alone \\
\hline PD8 J & 75 & M & AD & living with spouse \\
\hline PD9 J & 71 & M & FTD & living with spouse \\
\hline PD10 & 65 & M & Mixed & Living alone \\
\hline PD11 & 70 & $M$ & FTP & living with spouse \\
\hline PD12 & 61 & F & AD & living with spouse \\
\hline AD- Alzheimer's disease & & \\
\hline
\end{tabular}

VaD - Vascular dementia

FTD - Fronto-temporal dementia

Mixed - Mixed Alzheimer's and vascular dementia

$\mathrm{J}$ - Joint interview with the carer of the same number

\section{Themes}

The topics raised by participants during the interviews are under three main themes. These comprise a) fear of decline - the concern about a loss of skills caused by social distancing measures and efforts to avert decline; b) frustration concerning the dilemma of returning to normal - not feeling able to return to normal even when restrictions are eased, and c) neglect - disappointment about the lack of concern or consideration from individuals, groups, and authorities.

\section{Fear of decline}

There were some people with dementia had few concerns and enjoyed the quiet, and time to appreciate their surroundings during the pandemic, but the overarching feeling was that there were negative impacts on dementia-related symptoms and mood, related to not feeling able to engage in usual social and other 
activities. This incentivised people with dementia to attempt to avert decline and maintain positive by being busy. For carers, the additional responsibility was difficult, especially when noticeable decline and increased vulnerability altered their relationship.

Many people with dementia understood the importance of social contact and the value of keeping busy to maintain social skills and self-confidence to interact with others. A deterioration in abilities was understood to create difficulties for re-engaging with society, even when restrictions were eased.

PD10 my belief is that it was the dementia was accelerated on the communication side because of lack of practice. I wasn't doing things, I wasn't seeing people, not just the vocal side and the hearing side, but because of not being with people my almost body language reading skills deteriorated as well.

Whilst people with dementia spoke of their concern about their progression of dementia symptoms, some carers reported sadness relating to the changes in the skills and behaviour of the person with dementia and a realisation of the impact of the changes had on the relationship. People with dementia did not mention this. Other carers spoke of the positives of doing more activities together and valued a strong relationship that had helped them cope with the pressures of the pandemic. Being alone with the person with dementia was harder for those carers who felt their relationship had changed.

C9 I think it's just companionship, normal conversation and companionship, somebody just to... because [name's] lost his empathy and his interest in things, just somebody for me, do you know what I mean?

A change in the relationship was linked to increased responsibility to manage new risks relating to COVID safety and keeping the person with dementia occupied, but it could be challenging making sure there was enough to do.

C10 Obviously, it meant a bit more thinking ahead. So it was trying to find things that she was interested in. ...it was also keeping my wife engaged with it and not trying to do too much...

Although some enjoyed activities that they could do together, many carers felt lonely and bored, with limited opportunities to focus on their own well-being. As face-to-face support groups and services had stopped, both people with dementia and carers were isolated with no options for carer respite.

C4 he would go to the [redacted], which was the day centre; they'd come and pick up, which I paid for obviously, and they dropped him back, but it gave me six hours in which I could, um, do, you know! Without having to be watchful.

\section{Averting decline and maintaining positive mental health}

People with dementia experienced distress over expectations or experience of, decline, but wanted to ensure they spent time positively. People with dementia spoke about the importance of doing familiar activities that were interesting and pleasurable, but also new things. Several wanted to challenge themselves and practise skills they believed would be of benefit. This was purposeful and people with 
dementia found strength in realising they had been able to adapt and learn. For example joining online groups, doing mind training, learning names of people or objects when out, reading, and improving recognition of shape and colour.

P7 because of lockdown, I just got a 500-piece [jigsaw] but it was like... like brightly coloured and it... although it was hard to do I persevered with it.

P4 I realised that if I keep my brain active hopefully I can slow the process down of the disease

Being in contact with others was important to maintaining well-being. One element was altruism. One respondent (PD11) was determined to visit his friend in a care home and to continue to teach nursing students about dementia by using Zoom. This couple valued being NHS patient representatives:

C8 But of course, because we've both been at home, it's... it's just been a good thing for us. And it's really, having the NHS meetings that's... that's helped us.

PD8 It's helped us survive

For both people with dementia and carers with internet access, online social media was useful in the absence of face-to-face contact. This allowed meetings to continue, and in some cases increased contact with loved ones and support networks. However, those who did not have access to the internet may have missed out.

C1 so I've adapted that by ringing people what... when I need, well, ringing people on a regular basis really and so still maintaining contact but not as... not as much if I was on Skype maybe.

A commonly mentioned change during restrictions was increasing exercise levels. Three respondents with dementia talked extensively about their reasons for getting out for walks to manage their mental and physical well-being. One man enjoyed some chance social interaction whilst exploring his local woods:

PD6 l've met some very nice people, and it's... we were able to sort of have a brief talk and jokes and things like that, but still keeping our two metres apart, that sort of thing.

Another (PD1) enjoyed practising learning new walking routes and memorising names of people he met on his walks and was also 'quite proud' of improving his diabetes control through increased exercise. Another man (P10) was determined to lose weight and improve his mobility but to maintain distance from people he walked laps around his communal garden at night.

\section{Dilemma of returning to normal}

People with dementia experienced a dilemma in wanting to return to social activities but worsened dementia symptoms and continuing barriers within society made that hard, even with easing of social restrictions. The promise of vaccinations did not instil confidence.

PD6 is it ever going to get rid of coronavirus? 
They were wary about safety in the case of future easing of restrictions, and many struggled to remember or did not trust, the frequently-changing official information. Some had found it more comfortable to stay indoors.

PD4 I force myself to go out now. And I think this second lockdown is an excuse for me in a way that I can hide, that I don't want to go out much...

A loss of confidence in memory and remembering the rules were concerns for both people with dementia and carers, and were complicated by a more generalised feeling of anxiety about returning to mixing with people, thus prolonging their social isolation.

PD1 I'm very nervous, and still am of going outside the house. It's going into places where other people are. They creep up on you and accumulate round without even thinking. Because it is... it is very easy to... to not social distance when you're out because you tend to forget.

SP10 ...I'll say, 'Well it's because of the virus?' 'What virus?' But I just have to be very close to her all the time to make sure she's not doing something that causes a problem.

People felt nervous about going into health care settings during the pandemic but felt reassured by appropriate COVID-19 safety precautions when attending hospital and dental appointments. In contrast, there was some displeasure about the shift to telephone or online appointments. People wanted choice about the delivery of appointments so they could be confident the practitioner understood their personal needs. Some people with dementia wanted the option of having the carer present and a choice of telephone, online or face-to-face appointments (PD10). A carer (C5) described a long wait for a dementia review over the phone, but the person with dementia was contacted at very short notice, which was unsatisfactory because the carer needed to be present.

A change in the usual medical professional seen for dementia-related appointments caused some upset. People with dementia felt that without knowledge of their personal experiences of dementia, the impact of any changes in their dementia-related needs would not be well understood. Also, people with dementia wanted more information about what they could do during COVID-19 to remain independent and engage in meaningful activities for 'interaction and stimulation' (C10) but this was not being offered.

PD10 ...references I made to how I thought things had changed were dismissed in saying, "Well, you know, dementia does progress"...I wanted somebody to, kind of, give me coping strategies in a way, you know, almost like if... if it had been a physical thing, they'd give you exercises to do.

When talking about reduced social restrictions, some of the participants were uneasy because they thought members of the public would not understand their difficulties or vulnerability. One carer mentioned her support for a local initiative of wearing a lanyard to identify someone with dementia in health settings, but others had concerns about public perceptions of people with dementia: 
C9 I think you've got to be careful because I think once you start putting a label on them, you know, that they've got dementia they're open to abuse, aren't they?

To help people with essential needs, some high street adaptations had been helpful, such as a library providing a pick-up and drop-off point and a pharmacy delivering medicines to the car (PD2).

Another barrier encountered was a lack of understanding of the needs of people with dementia and carers when in shared public places.

C10 all the seating that had been provided there, most of it disappeared. And then the small amount of seating that was, was clearly labelled, 'Only for people with disabilities.'

This man summed up his feelings about the dilemma faced by people with dementia in getting back to normal:

P10 From a cosmetic point of view it was beneficial for the government to make us fearful so that we would stay in. Well, they've now got to spend millions undoing that...So that we feel like going out again.

\section{Neglect}

Not being contacted by individuals, groups, or authorities, not feeling included in official information provided, or in arrangements for easing restrictions, led to a feeling of neglect and exclusion. Two carers suggested that people with dementia were not given due consideration during lockdown in the same way as people with physical disabilities.

C10 It's a bit like obviously if you turn up at the door with someone in a wheelchair, it's obvious that they're disabled... the general public don't have much of an understanding of what difficulties you get with mental health problems, no matter what they are, but particularly with dementia.

Many felt surprised they had not been contacted. For example, two carers expressed disappointment that their church leader had not been in contact to offer emotional support. Others mentioned not being contacted by support services or medical professionals.

C2 I think there should have been some sort of communication to see if she was alright. And they used to come... so I'm a bit disappointed that they haven't done that this time, like, you know?

People did not tend to be proactive in asking for assistance but wanted to be asked about their wellbeing. By this stage in the pandemic, people had found solutions to meet their essential needs, but some felt the typical type of support offered was not what they needed.

C10 It never occurred to me to actually talk to people about it. Because you've then got to specify exactly what you want. I don't need physical support. I don't need any domestic support. But it's interaction and stimulation. 
Government information was found to be as confusing and non-specific to dementia and so people were not sure which rules were relevant. Carers also worried the person they cared for would not be able to follow the distancing or mask-wearing rules (C10, C4 and C2).

C6 I don't actually know which group we're supposed to be in, and what we're supposed to be doing and not doing.

Two people with dementia suggested there should be a central source for clear and trusted information, and somewhere to go in a time of crisis. Both people with dementia and carers thought that dementia networks were useful sources of information.

C4 [the] memory café is where, er, you tend to find out what's available, who to go to, who to speak to...You know, and you get that sort of thing.

There was also some disappointment about the lack of consultation regarding re-opening groups. One man who was caring for his wife with advanced dementia, for whom internet contact was not possible, understood the group could not continue day trips by bus, but had ideas about possible alternatives (such as a socially distanced walk); however, the service had not been in contact about re-opening.

C5 Talking to fellow caregivers, I think we would have been far more bold, actually... Because of the benefit, both to me and to [redacted] I feel that she gets from the group, if they could have found some way of bringing them together...

\section{Discussion}

This study explored the impact of social distancing measures on people with dementia and carers living in the community during restrictions and before the COVID-19 vaccination roll-out in England and Wales. We identified three interrelated experiential themes that add to the existing literature focussing on earlier experiences of the pandemic: fear of decline, the dilemma of returning to normal, and neglect. Many of the interviewees found ways to adapt their behaviour to stay positive and help prevent a decline in capabilities or mood, but some experienced reduced confidence with a decline in capabilities. This created a cyclical dilemma that made returning to usual activities difficult even during periods of reduced restrictions. Re-engaging with society was challenging due to feeling less capable, or feeling that different aspects of society had not adjusted adequately to be inclusive. Knowledge of the upcoming vaccination programme did not instil feelings of hope. These experiences related to several domains relevant to social exclusion (Walsh et al 2017), in particular services, amenities and mobility, social relations, and community. The impact of this, although relevant to the older population, was exacerbated by the challenges of living with dementia or caring for a person with dementia.

In this study, cultural, social, and emotional dimensions of exclusion impacted heavily on both people with dementia and carers during the pandemic because meaningful social connection both at a personal and a civic level was important for self-worth and stimulation to help avert dementia progression. There 
was less evidence to show that people felt excluded at an economic or environmental level. These findings support evidence that the quality of relationships and opportunities to socialise are pivotal elements of social inclusion for people with dementia (Pinkert et al., 2021. Quinn et al. 2021a, Quinn et al. 2021b). Social relations are a dimension of social exclusion in older age (Walsh et al. 2017) but the reduction in or loss of skills in communication during the pandemic for people with dementia could lead to further isolation and restriction of opportunities for social relations and civic engagement. In addition, for carers, increased practical caring responsibilities and dealing with behavioural issues are known to impact the relationship with the care recipient (Quinn et al. 2009), potentially furthering the experience of isolation. Others have found the additional stress caused by having to balance COVID risk with well-being added to carers' responsibilities (Cagnin et al., 2020). In some cases, the relationship between the carer and the person with dementia seemed to strengthen by spending more time together, but for others, the relationship with the person with dementia changed with a rapid increase in responsibility and care needs.

The experience of decline in abilities for the person with dementia and greater caring responsibilities for carers led to increased vulnerability to exclusion. Increased responsibility meant reduced opportunities for carers to have social interaction with others or to have respite when things reopened. For people with dementia, loss of communication skills and confidence made interaction with friends and family, and the wider community, more problematic and possibly irretrievable. Experiences or fears of stigma reported widely pre-pandemic (Herrmann et al., 2018) were alluded to and are likely to have been worsened due to reduced capabilities or fears about loss of capabilities.

Both people with dementia and carers as individuals or dyads felt they were left to deal with the consequences of the immediate or longer-term implications of the pandemic but found their own solutions to manage as best as they could, often changing behaviours and adapting. Although personal strategies were useful, and some may have felt comfortable with reduced social contact, there was little evidence of specific dementia support at a broader social or cultural level. There was little evidence of the experience of decline being acknowledged or understood outside of individual experience, indicating social exclusion at the meso- (interactional environment) and macro- (broader social) level (Pinkert et al., 2021).

The dilemma of people with dementia and carers wanting to reengage with society but being restricted by changes in dementia symptoms and feelings of increased vulnerability adds understanding to the 'complex health issues' (Pinkert et al., 2021) causing micro-level social exclusion. Both people with dementia and carers experienced exclusion via a lack of consultation about returning to groups or services. Official COVID-19 information was neither clear nor specific to their situation. The consequences of reduced social interaction have been reported by both people with dementia and carers elsewhere (Talbot and Briggs 2021, Giebel et al., 2020, O'Rourke et al. 2021b) and are anecdotally supported in online forums such as www.dementiadiaries.org but our data show this was not addressed as the pandemic progressed. 
The overarching feeling of neglect amongst people with dementia and carers during the pandemic was identified in our earlier study conducted in the first wave of the pandemic (O'Rourke et al., 2021b). There is a wider and ongoing problem of social exclusion affecting people with dementia, but this has almost doubled during the pandemic (Cohen et al., 2020), and the lack of hope to move forward out of the pandemic is a cause for concern. A general feeling of needs not being understood could have contributed to low expectations based on experience. To address the consequences of pandemic restrictions, and possibly to gain control, individuals adapted to cope, a process also seen previously (O'Rourke et al. 2021b). Accessing online social activities, groups and information was a source of comfort for some, but also a source of exclusion for those unable to participate. Where inclusion was promoted this was demonstrated through individual examples of localised access to goods and services. These local adaptations were not dementia-specific but helped people to go about their usual activities and may have helped them feel included.

The findings add understanding of the consequences of ongoing social restrictions in deepening the preexisting risk of social exclusion amongst people with dementia and have implications for a return to usual social activities. This study offers some insights into the ongoing needs of both people with dementia and carers despite the promise of vaccinations. People were recruited from across England and Wales to take into account of differences in local rules. To collect and analyse the data promptly we did not set out to analyse the sample according to sub-groups based on age and ethnicity or gender, nor did we recruit large enough numbers to allow this. Although we did not identify evidence of social exclusion linked to an economic or environmental dimension, a larger sample could uncover other exclusionary impacts during the pandemic.

People with dementia and carers identified what was important to them and attempted to mitigate the impacts of social isolation, but more help was needed. There was a lack of proactive contact with people with dementia from health and social care or council-based services, and although some 'checking in services' to see how people were and to signpost to support were appreciated earlier on in the pandemic (O'Rourke et al. 2021b), these did not continue for our participants. While much of society had enjoyed the easing of restrictions at various stages over the preceding summer months our group had not felt able to re-engage in the same way as other members of the public. In the context of moving to postpandemic 'recovery' with more people vaccinated, a focus on better respite for carers and on rebuilding confidence and rehabilitation for people with dementia may be worthwhile. The difficulties in accessing or using the internet to find information and services should be acknowledged and alternative methods for keeping people in contact and informed could be explored. There is a need to offer a range of options for accessing health and social care rather than relying too heavily on telephone- or internet-based appointments for people who may find these forms of communication difficult (Kalicki et al. 2021); the shift to online groups and services was initially welcomed but the longer-term effects are not yet evaluated. Alongside this, governments and policy-makers could do more to include the voices of people with dementia in finding ways of allowing them to feel safe accessing the shops, services, and entertainment that normally form part of their lives. 


\section{Conclusion}

Qualitative data collected during the pandemic has uncovered the need for people with dementia and carers to feel part of normal society during periods of lockdown and this study offers some solutions for the future. Although people demonstrated problem-solving ability and resilience, the pandemic appeared to have exacerbated the extent of social exclusion and there was little expectation of external intervention. People with dementia and carers needed practical advice about what they could do during the pandemic to stay actively and meaningfully engaged, and carers experiencing additional demands needed practical solutions to enable them to gain respite. These findings offer guidance about supporting people with dementia and carers during future periods of pandemic-related social restriction.

\section{Declarations}

\section{ACKNOWLEDGEMENTS}

We acknowledge the support of NIHR Dementias and Neurodegeneration Specialty (DeNDRoN), and Health and Care Research Wales with IDEAL cohort recruitment and data collection. We gratefully acknowledge the local principal investigators and researchers involved in participant recruitment and assessment within these networks. We are grateful to the IDEAL study participants for their participation in the IDEAL and INCLUDE studies, to the wider group of IDEAL programme researchers, and to members of the ALWAYs group and the Project Advisory Group for their support.

\section{FUNDING}

'Identifying and mitigating the individual and dyadic impact of COVID-19 and life under physical distancing on people with dementia and carers (INCLUDE)' was funded by the Economic and Social Research Council (ESRC) through grant ES/V004964/1. Investigators: Clare, L., Victor, C., Matthews, F., Quinn, C., Hillman, A., Burns, A., Allan, L., Litherland, R., Martyr, A., Collins, R., \& Pentecost, C. ESRC is part of UK Research and Innovation (UKRI).

'Improving the experience of Dementia and Enhancing Active Life: living well with dementia. The IDEAL study' was funded jointly by the Economic and Social Research Council (ESRC) and the National Institute for Health Research (NIHR) through grant ES/L001853/2. Investigators: L. Clare, I.R. Jones, C. Victor, J.V. Hindle, R.W. Jones, M. Knapp, M. Kopelman, R. Litherland, A. Martyr, F.E. Matthews, R.G. Morris, S.M. Nelis, J.A. Pickett, C. Quinn, J. Rusted, J. Thom. ESRC is part of UK Research and Innovation (UKRI). IDEAL data were deposited with the UK data archive in April 2020 and will be available to access from April 2023. Details of how the data can be accessed after that date can be found here: http://reshare.ukdataservice.ac.uk/854293/

'Improving the experience of Dementia and Enhancing Active Life: a longitudinal perspective on living well with dementia. The IDEAL-2 study' is funded by Alzheimer's Society, grant number 348, AS-PR2-16- 
001. Investigators: L. Clare, I.R. Jones, C. Victor, C. Ballard, A. Hillman, J.V. Hindle, J. Hughes, R.W. Jones, M. Knapp, R. Litherland, A. Martyr, F.E. Matthews, R.G. Morris, S.M. Nelis, C. Quinn, J. Rusted.

L. Clare and L Allan acknowledge support from the NIHR Applied Research Collaboration South-West Peninsula.

The views expressed are those of the author(s) and not necessarily those of the ESRC, UKRI, NIHR, the Department of Health and Social Care, the National Health Service, or Alzheimer's Society. The support of ESRC, NIHR and Alzheimer's Society is gratefully acknowledged.

\section{CONFLICT OF INTEREST}

We have no conflict of interest to declare.

\section{References}

1. Alzheimer's Society (2020). Worst Hit: dementia during coronavirus. Alzheimer's Society. https://www.alzheimers.org.uk/sites/default/files/2020-09/Worst-hit-Dementia-during-coronavirusreport.pdf

2. Bacsu, J. D., O'Connell, M. E., Cammer, A., Azizi, M., Grewal, K., Poole, L., Green, S., Sivananthan S., Spiteri, R. J. (2021). Using twitter to understand the COVID-19 experiences of people with dementia: Infodemiology Study. Journal of Medical Internet Research, 23(2), e26254. http://doi.org/10.2196/26254

3. Borelli, W. V., Augustin, M. C., de Oliveira, P. B. F., Reggiani, L. C., Bandeira-de-Mello, R. G., SchumacherSchuh, A. F., Chaves, M.L.F., Castilhos, R. M. (2021). Neuropsychiatric symptoms in patients with dementia associated with increased psychological distress in caregivers during the COVID-19 Pandemic. Journal of Alzheimer's Disease, 80(4), 1705-1712. http://doi.org/10.3233/JAD-201513

4. Cagnin, A., Di Lorenzo, R., Marra, C., Bonanni, L., Cupidi, C., Lagana, V., Rubino, E., Vacca, A., Provero, P., Isella, V., Vanacore, N., Agosta, F., Appollonio, I., Caffarra, P., Pettenuzzo, I., Sambati, R., Quaranta, D., Guglielmi, V., Logroscino, G., Filippi, M., Tedeschi, G., Ferrarese, C., Rainero, I., Bruni, A.C INdem COVID-19 Study Group. (2020). Behavioral and psychological effects of coronavirus disease-19 quarantine in patients with dementia. Frontiers in Psychiatry, 11, 578015.

http://doi.org/10.3389/fpsyt.2020.578015

5. Canevelli, M., Valletta, M., Toccaceli Blasi, M., Remoli, G., Sarti, G., Nuti, F., Sciancalepore, F., Ruberti, E., Cesari, M., Bruno, G. (2020). Facing dementia during the COVID-19 outbreak. Journal of the American Geriatrics Society, 68(8) 1673-1676. http://doi.org/10.1111/jgs.16644

6. Carpinelli Mazzi, M., lavarone, A., Musella, C., De Luca, M., de Vita, D., Branciforte, S., Coppola, A., Scarpa, R., Raimondo, S., Sorrentino, S., Lualdi, F., Postiglione, A. (2020). Time of isolation, education and gender influence the psychological outcome during COVID-19 lockdown in caregivers of patients with dementia. European Geriatric Medicine, 11(6), 1098. http://doi.org/10.1007/s41999-020-00413- 
7. Clare, L., Martyr, A., Gamble, L., Pentecost, C., Collins, R., Dawson, E., Hunt, A., Parker, S., Allan, L., Burns, A. (2021). Impact of COVID-19 on 'living well' with mild-to-moderate dementia in the community: findings from the IDEAL cohort. Journal of Alzheimer's Disease. 1-16 http://doi.org/10.3233/JAD-215095

8. Clare, L., Nelis, S. M., Quinn, C., Martyr, A., Henderson, C., Hindle, J. V., Jones., lan R., Jones, R.W., Knapp, M., Kopelman, M. D. (2014). Improving the experience of dementia and enhancing active lifeliving well with dementia: study protocol for the IDEAL study. Health and Quality of Life Outcomes, 12(1), 1-15. http://doi.org/10.1186/s12955-014-0164-6.

9. Clemente-Suárez, V. J., Navarro-Jiménez, E., Jimenez, M., Hormeño-Holgado, A., Martinez-Gonzalez, M. B., Benitez-Agudelo, J. C., Perez-Palencia, N., Laborde-Cárdenas, C.C., Tornero-Aguilera, J. F. (2021). Impact of COVID-19 pandemic in public mental health: an extensive narrative review. Sustainability, 13(6), 3221. <background-color:\#CFBFB1;uverticalalign:super;>https://doi.org/10.3390/su13063221</background-color:\#CFBFB1;uverticalalign:super;>

10. Cohen, G., Russo, M. J., Campos, J. A., \& Allegri, R. F. (2020). COVID-19 epidemic in Argentina: Worsening of behavioral symptoms in elderly subjects with dementia living in the community. Frontiers in Psychiatry, 11, 866. http://doi.org/10.3389/fpsyt.2020.00866

11. Gale, N. K., Heath, G., Cameron, E., Rashid, S., \& Redwood, S. (2013). Using the framework method for the analysis of qualitative data in multi-disciplinary health research. BMC Medical Research Methodology, 13(1), 1-8. https://doi.org/10.1186/1471-2288-13-117

12. Gardiner, C., Geldenhuys, G., \& Gott, M. (2018). Interventions to reduce social isolation and loneliness among older people: an integrative review. Health and Social Care in the Community, 26(2), 147-157. http://doi.org/10.1111/hsc. 12367

13. Giebel, C., Cannon, J., Hanna, K., Butchard, S., Eley, R., Gaughan, A., Komuravelli, A., Shenton, J., Callaghan, S., Tetlow, H., Limbert, S., Whittington, R., Rogers, C., Rajagopal, M., Ward, K., Shaw, L., Corcoran, R., Bennett, K., Gabbay, M. (2020). Impact of COVID-19 related social support service closures on people with dementia and unpaid carers: a qualitative study. Aging and Mental Health, 25(7), 1281-1288. http://doi.org/10.1080/13607863.2020.1822292

14. Giebel, C., Hanna, K., Callaghan, S., Cannon, J., Butchard, S., Shenton, J., Komuravelli, A., Limbert, S., Tetlow, H., Rogers, C., Eley, R., Rajagopal, M., Ward, K., Gabbay, M. (2021a). Navigating the new normal: accessing community and institutionalised care for dementia during COVID-19. Aging and Mental Health, 28, 1-6. http://doi.org/10.1080/13607863.2021.1914545

15. Giebel, C., Lord, K., Cooper, C., Shenton, J., Cannon, J., Pulford, D., Shaw, L., Gaughan, A., Tetlow, H., Butchard, S., Limbert, S., Callaghan, S., Whittington, R., Rogers, C., Komuravelli, A., Rajagopal, M., Eley, R., Watkins, C., Downs, M., Gabbay, M. (2021b). A UK survey of COVID-19 related social support closures and their effects on older people, people with dementia, and carers. International Journal of Geriatric Psychiatry, 36(3), 393-402. http://doi.org/10.1002/gps.5434 
16. Greenwood, N., Mezey, G., \& Smith, R. (2018). Social exclusion in adult informal carers: A systematic narrative review of the experiences of informal carers of people with dementia and mental illness. Maturitas, 112, 39-45. http://doi.org/10.1016/j.maturitas.2018.03.011

17. Herrmann, L. K., Welter, E., Leverenz, J., Lerner, A. J., Udelson, N., Kanetsky, C., \& Sajatovic, M. (2018). A systematic review of dementia-related stigma research: can we move the stigma dial? American Journal of Geriatric Psychiatry, 26(3), 316-331. http://doi.org/10.1016/j.jagp.2017.09.006

18. Ismail, II., Kamel, W. A., \& Al-Hashel, J. Y. (2021). Association of COVID-19 pandemic and rate of cognitive decline in patients with dementia and mild cognitive impairment: A cross-sectional study. Gerontology and Geriatric Medicine, 7, 1-7. http://doi.org/10.1177/23337214211005223

19. Kalicki, A. V., Moody, K. A., Franzosa, E., Gliatto, P. M., \& Ornstein, K. A. (2021). Barriers to telehealth access among homebound older adults. Journal of the American Geriatrics Society. 69(9), 24042411. http://doi.org/10.1111/jgs.17163

20. Kane, M., \& Cook, L. (2013). Dementia 2013: The hidden voice of loneliness. Alzheimer's Society. https://www.alzheimers.org.uk/dementia2013

21. Khan, S., Combaz, E., \& McAslan Fraser, E. (2015). Social exclusion: topic guide. Revised Edition. GSDRC, University of Birmingham.

22. Litherland, R., Burton, J., Cheeseman, M., Campbell, D., Hawkins, M., Hawkins, T., Oliver, K., Scott, D., Ward, J., Nelis, S. M., Quinn, C., Victor, C., Clare, L. (2018). Reflections on PPI from the 'Action on Living Well: Asking You' advisory network of people with dementia and carers as part of the IDEAL study. Dementia, 17(8), 1035-1044. http://doi.org/10.1177/1471301218789309

23. Masterson-Algar P., Cheshire Allen, M., Hyde, M., Keating, N., \& Windle, G. (2021). Exploring the impact of COVID-19 on the care and quality of life of people with dementia and their carers: A scoping review. Dementia, 1-29. http://doi.org/10.1177/14713012211053971

24. O'Rourke, G. J., Pentecost, C., van den Heuvel, E., Victor, C., Quinn, C., Hillman, A., Litherland, R., Clare, L. (2021a). Living with dementia during the COVID-19 pandemic: coping and support needs of community dwelling people with dementia and their family carers. Research findings from the IDEAL COVID-19 Dementia Initiative (IDEAL-CDI). NIHR Older People and Frailty Policy Research Group. https://documents.manchester.ac.uk/display.aspx?DoclD =54837

25. O'Rourke, G. J., Pentecost, C., van den Heuvel, E., Victor, C., Quinn, C., Hillman, A., Litherland, R., Clare, L. (2021b). Living with dementia under COVID-19 restrictions: coping and support needs among people with dementia and carers from the IDEAL cohort. Aging and Society. 1-23. http://doi.org/10.1017/S0144686X21001719

26. Pinkert, C., Köhler, K., von Kutzleben, M., Hochgräber, I., Cavazzini, C., Völz, S., Palm, R., Holle, B. (2021). Social inclusion of people with dementia-an integrative review of theoretical frameworks, methods and findings in empirical studies. Ageing and Society. 41(4), 773-793. http://doi.org/10.1017/S0144686X19001338

27. Pongan, E., Dorey, J. M., Borg, C., Getenet, J. C., Bachelet, R., Lourioux, C., Laurent, B. Ray, R., Rouch, I. (2021). COVID-19: Association between increase of behavioral and psychological symptoms of 
dementia during lockdown and caregivers' poor mental health. Journal of Alzheimer's Disease, $80(4)$, 1713-1721. http://doi.org/10.3233/JAD-201396

28. Rochford-Brennan, H., \& Keogh, F. (2020). Giving voice to those directly affected by the COVID-19 pandemic - the experience and reflections of a person with dementia [version 2; peer review: 3 approved]. HRB Open Research, 3(29). http://doi.org/10.12688/hrbopenres.13063.2

29. Seifert, A., Cotten, S. R., \& Xie, B. (2021). A double burden of exclusion? Digital and social exclusion of older adults in times of COVID-19. The Journals of Gerontology: Series B, 76(3), e99-e103. http://doi.org/10.1093/geronb/gbaa098

30. Simonetti, A., Pais, C., Jones, M., Cipriani, M. C., Janiri, D., Monti, L., Landi, F., Bernabei, R., Liperoti, R., Sani, G. (2020). Neuropsychiatric symptoms in elderly with dementia during COVID-19 pandemic: Definition, treatment, and future directions. Frontiers in Psychiatry, 11, 579842. http://doi.org/10.3389/fpsyt.2020.579842

31. Talbot, C. V., \& Briggs, P. (2021). 'Getting back to normality seems as big of a step as going into lockdown': the impact of the COVID-19 pandemic on people with early to middle stage dementia. Age and Ageing, 50(3), 657-663. doi: 10.1093/ageing/afab012

32. Walsh K., Scharf, T. Keating, N. (2017). Social exclusion of older persons: a scoping review and conceptual framework. European Journal of Ageing, 14(1) 91-98. http://doi.org/10.1007/s10433016-0398-8

33. Wright, T., \& O'Connor, S. (2018). Reviewing challenges and gaps in European and global dementia policy. Journal of Public Mental Health, 17(4), 157-167. https://doi.org/10.1108/JPMH-02-20180012

34. Quinn, C., Clare, L. Woods, B. (2009). The impact of quality of relationship on the experiences and wellbeing of caregivers of people with dementia: A systematic review. Aging and Mental Health, 13(2), 143-154. http://doi.org/10.1080/13607860802459799

35. Quinn, C., Hart, N., Henderson, C., Litherland, R., Pickett, J., Clare, L. (2021a). Developing supportive local communities: Perspectives from people with dementia and caregivers participating in the IDEAL programme. Journal of Aging \& Social Policy, 1-21. http://doi:10.1080/08959420.2021.1973341

36. Quinn, C., Pickett, J. A., Litherland, R., Morris, R. G., Martyr, A., Clare, L., On behalf of the IDEAL Programme Team. (2021b). Living well with dementia: What is possible and how to promote it. International Journal of Geriatric Psychiatry. http://doi:10.1002/gps.5627

37. Wu, Y.-T., Clare, L., Jones, I.R., Martyr, A., Nelis, S.M., Quinn, C., Victor, C.R., Lamont, R.A., Rippon, I., Matthews, F.E., On behalf of the Improving the experience of Dementia and Enhancing Active Life (IDEAL) study. (2018). Inequalities in living well with dementia - the impact of deprivation on wellbeing, quality of life and life satisfaction: results from the Improving the experience of Dementia and Enhancing Active Life study. International Journal of Geriatric Psychiatry, 33, 1736-1742. http://doi: 10.1002/gps.4998 